\title{
The Phenomena of Amplification in 2D Photonic Macroporous Silicon
}

\author{
Lyudmyla A. Karachevtseva \\ V. Lashkariov Institute of Semiconductor Physics of NAS of Ukraine; \\ 45 Nauky Ave., Kyiv-03028, Ukraine
}

Effects of increase in absorption of electromagnetic radiation, amplification of photoconductivity and Raman scattering by $2 D$ structures of macroporous silicon were investigated. Maximal photoconductivity was measured (1) at normal incidence of electromagnetic radiation, (2) at a critical angle concerning macropore walls and (3) at a grazing angle concerning a surface of structure. Angular dependences of photoconductivity, as well as enhancement of the photoconductivity in comparison with monocrystal silicon, primary absorption p-component of electromagnetic radiation testified to formation of surface electromagnetic waves in illuminated macrporous silicon structures. Its effects result in amplification of a local electric field on a surface of macroporous silicon structure and a macropore surface. Electric components of an electromagnetic field on macropore surface are transferred in volume to distance about $l_{e}$. Shift of a spectrum maximum in the long-wavelength party is observed at the maximal amplification of photoconductivity. The measured built-in electric field on a macropore surface achieves $10^{6} \mathrm{~V} / \mathrm{cm}$, the signal of photoconductivity amplifies $10^{2}$ times, and Raman scattering - up to one order of value.

\section{Introduction:}

Photonic crystals are periodic structures with modulated dielectric constant. The spectrum of electromagnetic radiation is divided by zones, forbidden for its propagation similar to electronic bands in semiconductors. The principal works with the idea of photonic band gap formation were published in $1987[1,2]$. The spectrum of the application use of the photon localisation effect is wide: filters, reflectors and substrates for antennas in the microwave range; wave guides which correct the distribution of light in optical fibre; zero-threshold lasers and low-loss resonators; electromagnetic wave filters. The effect of the photonic band gap formation opens the perspectives of the manufacture of photon analogue of the semiconductor devices.

lakar@isp.kiev.ua 
Effects of the enhancement by periodic structures of illumination and absorption of electromagnetic radiation, Raman scattering and light transformation in a photocurrent were intensively investigated during last decades [3, 4]. The basic mechanism of the specified effects is formation of modes of the surface oscillator fluctuations (surface electromagnetic waves or surface polaritons).

Resonant amplification of an electromagnetic field on border of the surface-active environment at surface polariton excitation results in increase of a photocurrent on the surface-barrier structures. Therefore surface polaritons find the increasing application in photodetectors (thin-film ones and with the narrow surface active area). In the metal-semiconductor, metal-dielectric-semiconductor structures the concentration of energy of electron-hole pairs on a surface is important, which reduces recombination in the region of a volume charge. At present in connection with the intensive development of a new direction of solid state physics - the photonic crystals, the new impulse was received by investigations of the surface waves in the periodic structures with frequencydependent dielectric permeability. In the given paper results of researches of effects of increase in absorption of electromagnetic radiation and amplification of photoconductivity in 2D structures of macroporous silicon are resulted.

\section{Methodology:}

The most effective methods of photonic band gap formation are lithographic methods with the electrochemical procedure. Such methods permit to fabricate semiconductor periodical structures from nanometer to micron scale. Especially, the method of light assisted electrochemical etching permits to fabricate microporous (pore width $<2 \mathrm{~nm}$ ), mesoporous (pore with width 2$50 \mathrm{~nm}$ ) or macroporous (pore width $>50 \mathrm{~nm}$ ) structures depending upon substrate resistivity and anodization conditions. The photonic band gaps based on disodered dielectric structures open the door to a very large scale of the inexpensive materials. Such promising material for the 2D photonic structure formation is macroporous silicon.

The starting material consisted of n-type silicon with $\langle 100\rangle$ orientation and 2-5 $\Omega . \mathrm{cm}$ resistivity. Macropores of 1-15 micron diameters were formed due to the generation and transfer of nonequilibrium holes to the $n$-Si electrochemically treated surface as a result of the optical band-to-band electron-hole generation $[5,6]$. Initial $\mathrm{n}$-Si plates were chemically polished in 1:3 $\mathrm{HF}$ and $\mathrm{HNO}_{3}$ solution and anisotropy etched in $10 \%$ solution of $\mathrm{KOH}$. An ohmic contact was provided by rubbing In-Ga eutectic around the circular area. The samples were mounted in an electrochemical cell and connected to 
potentiostat by the 3 -electrode scheme. The electrolyte was 5 weight percent hydrofluoric acid. The applied voltage was measured in relation to a platinun wire near the sample surface. During the electrocheemical etching process the samples were illuminated by a radiation from a $100 \mathrm{~W}$ tungsten lamp. Periodic structures and structures with arbitrary distribution of macropores were fabricated (Fig. 1).

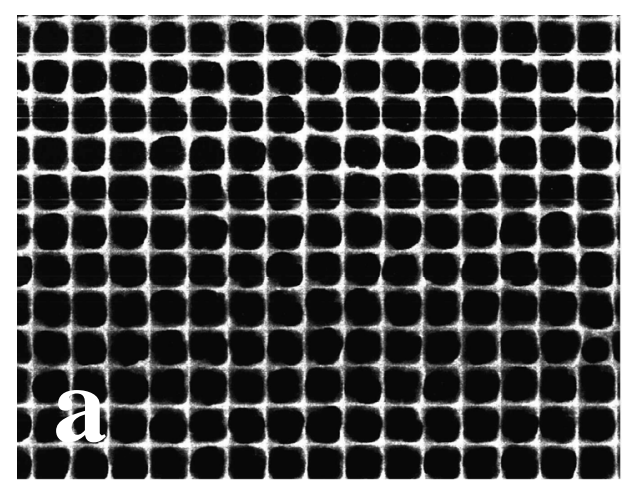

$20 \mu \mathrm{m}$

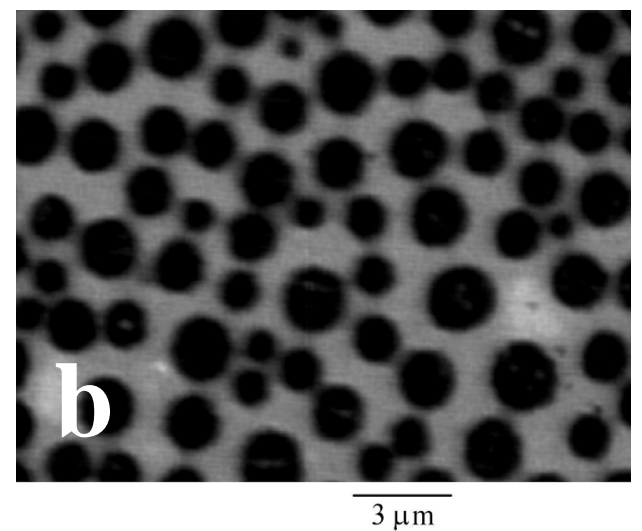

$3 \mu \mathrm{m}$

Fig. (1): Two dimensional photonic structures of macroporous silicon with periodical (a) and arbitrary (b) macropore distribution.

Optical transmittance was measured using IR Fourier spectrometer IFS113, IR spectrophotometer Spicord-85 and He-Ne laser. Absorption coefficient $\alpha$ was recalculated taking into account the measured absorbance value $I_{0} / I_{\mathrm{ps}}\left(I_{0}\right.$ incident irradiation, $\mathrm{I}_{\mathrm{ps}}$ - transmitted irradiation) and the adsorbance value of reference silicon plates $\mathrm{I}_{0} / \mathrm{I}_{\mathrm{Si}}\left(\mathrm{I}_{\mathrm{Si}}\right.$ - irradiation intensity, transmitted through reference silicon plate). Measurements of the Raman spectra were carried out at room temperature with the help of DFS-24 spectrometer.

Indium contacts to monocrystalline and to macroporous silicon were formed by vacuum deposition of metal and temperature annealing. Thermal annealing of the "In/n-Si" and "In/ macroporous n-Si" structures was performed at temperature of $200-500^{\circ} \mathrm{C}$. Without annealing the volt-ampere characteristics of the "indium/silicon" contacts and of the "indium/macroporous silicon" contacts are nonlinear and are similar to the volt-ampere characteristics of metal/dielectric/semiconductor structures. This is due to a layer of oxide. At higher annealing temperatures the oxide potential barrier was lowered and at an annealing temperature of $\mathrm{T}=400^{\circ} \mathrm{C}$ in an atmosphere of hydrogen the voltampere characteristics of both types of structures were linear. At higher annealing temperatures the effect of overcompensation of contact area by 
indium took place, and the linearity of the volt-ampere characteristics was broken. With a change in annealing temperature from $200^{\circ} \mathrm{C}$ up to $400^{\circ} \mathrm{C}$ the transient contact resistance decreased $2-2.5$ times and reached an optimal value of $R_{0} \approx 4-10 \Omega \cdot \mathrm{cm}^{2}$, that is less, than the transient resistance of an Alporous silicon contact, formed at a temperature of $\mathrm{T}=400^{\circ} \mathrm{C}[7,8]$.

\section{Results and discussion}

\subsection{Optical transmittance.}

Absolute photonic band gap was measured at wavelengths between one and two optical periods $\left(\lambda_{\mathrm{a}}<\lambda<2 \lambda_{\mathrm{a}}\right)$ of macroporous silicon structure (Fig. 2). Obtained results correlate qualitatively with calculations $[9,10]$. In twodimensional periodic structure with period $a$ absolute photonic band gap forms at $\lambda=1.2-1.5 \lambda_{a}\left(\lambda_{a}-\right.$ is the optical period of the photonic structure, equaled to $\left.\left(a-D_{p}\right) \varepsilon^{l / 2}+D_{p}\right)$. Absolute band gap below the first band was not measured. So macroporous silicon structures are effective media for long electromagnetic wave [11].

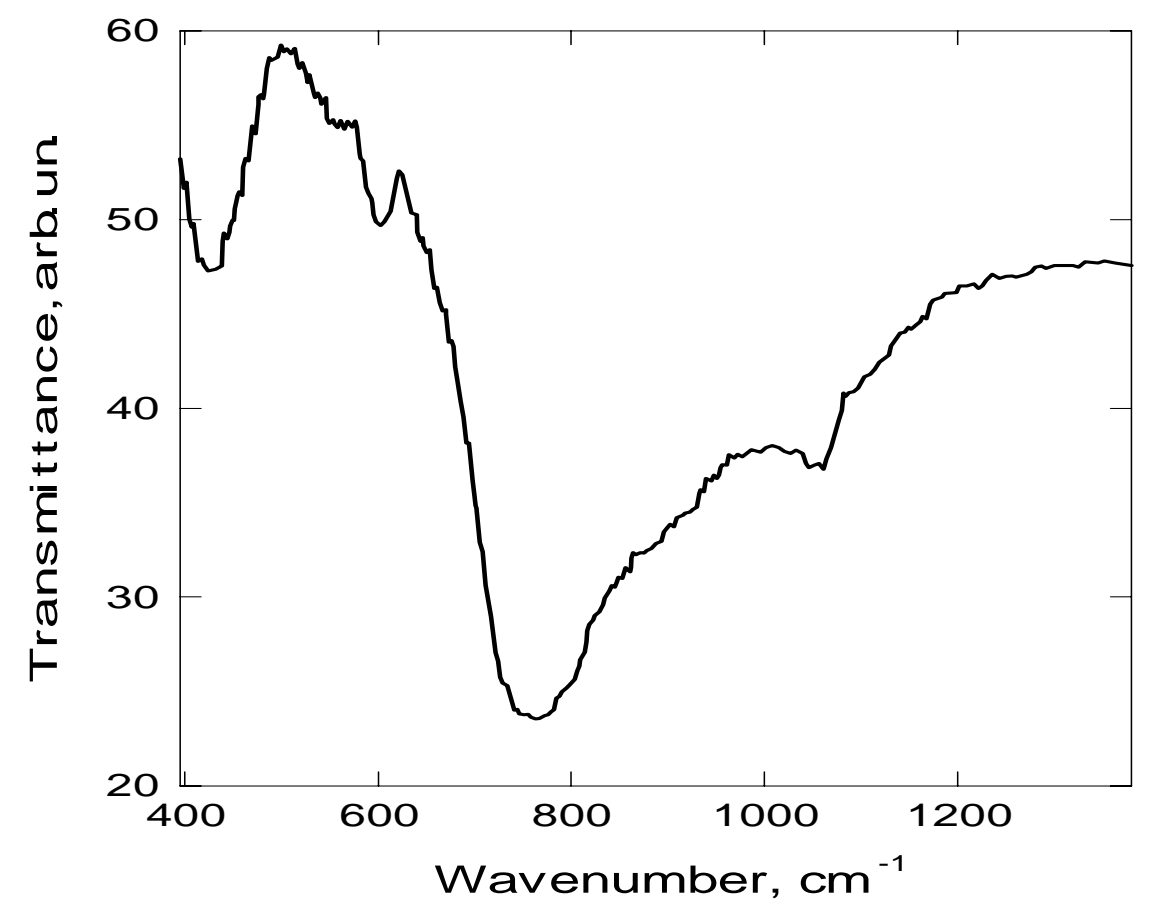

Fig. (2): Transmittance of electromagnetic waves propagated in 2D macroporous silicon structure parallel to macropores. 
Absorption spectra of macroporous silicon with different pore diameters and concentration have common features at $\lambda \leq \lambda_{a}$ (Fig. 3a). There is an essential reduction in transmitttance of electromagnetic radiation with a shortening of the wavelength. Recalculated absorption coefficient is equaled $300-500 \mathrm{~cm}^{-1}$, as is shown on (Fig.3b).

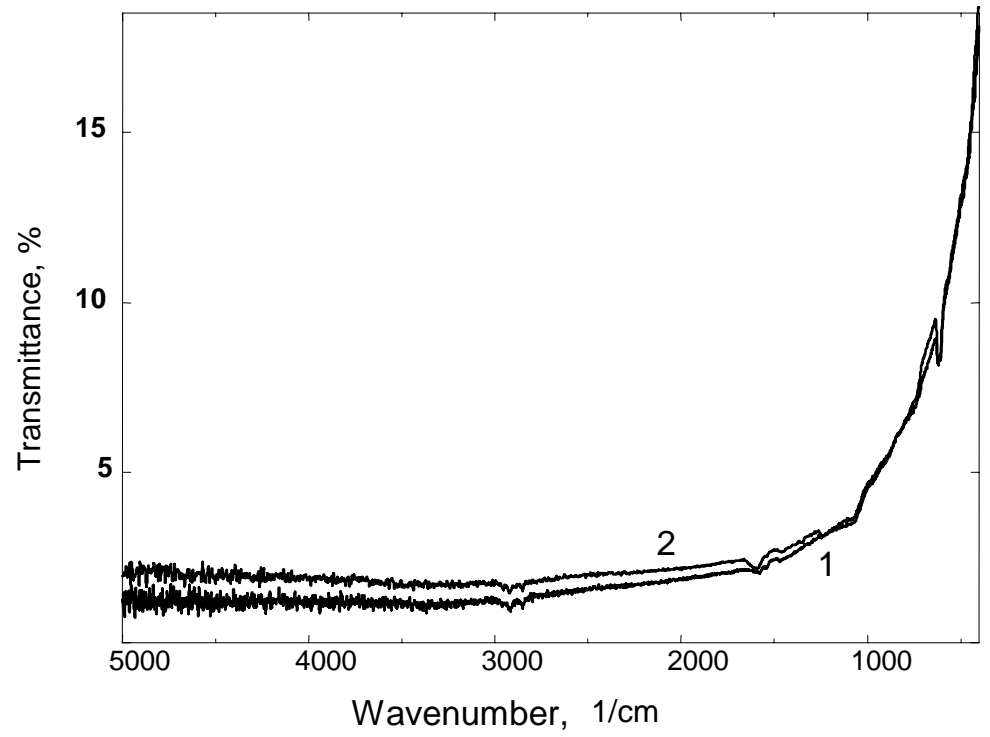

Fig. (3a): Transmittance spectra of two macroporous silicon structures.

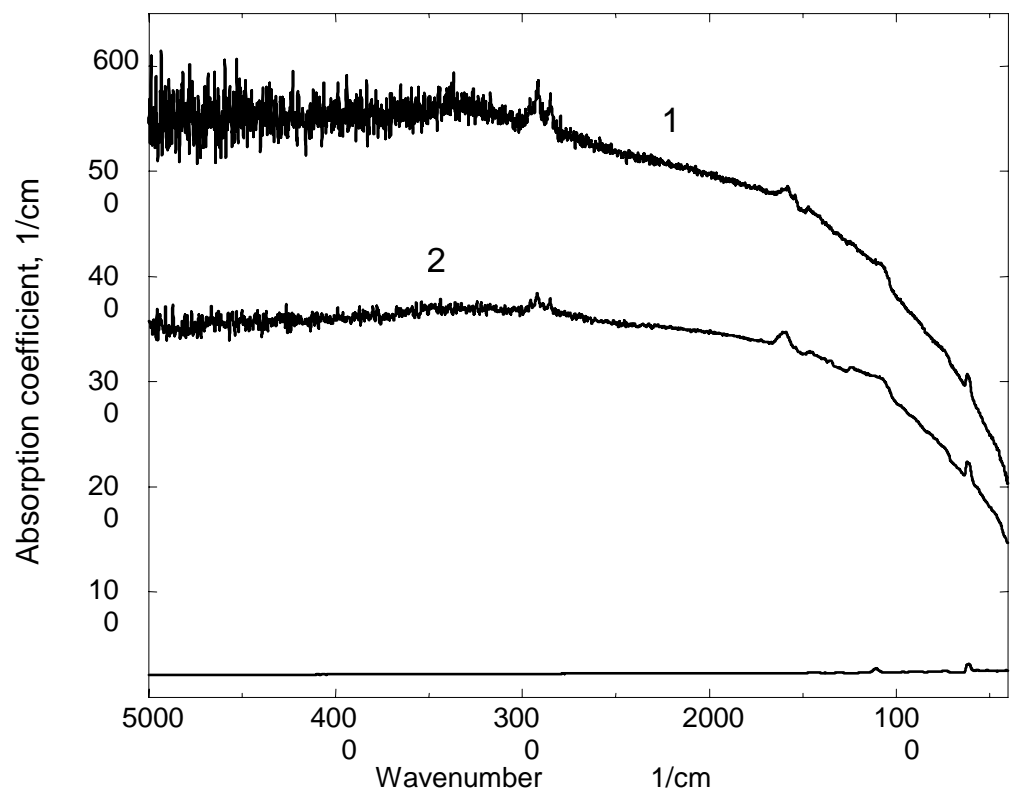

Fig. (3b): Spectral distribution of the effective absorption coefficient. 
Optical transmittance of the macroporous structure was $3-6 \cdot 10^{-3}$ apparently homogeneous material [12] accordingly to measurements at $\lambda=1.15$ $\mu \mathrm{m}$ and $\lambda=3.39 \mu \mathrm{m}$ of He-Ne laser. At short wavelengths $\lambda<D_{p}$ the optical transmittance slightly grows. Transmission spectra contain steps, as in (Fig. 4). The step frequency in the long-wave part of the spectrum is proportional to the distance between the pores $\left(\Delta v^{1} \sim a-D_{p}\right.$, Figure 5a), and in the short-wave region it is proportional to the diameter of the pores $\left(\Delta \mathrm{v}^{\mathrm{s}} \sim D_{p}\right.$, Fig. $\left.5 \mathrm{~b}\right)$.

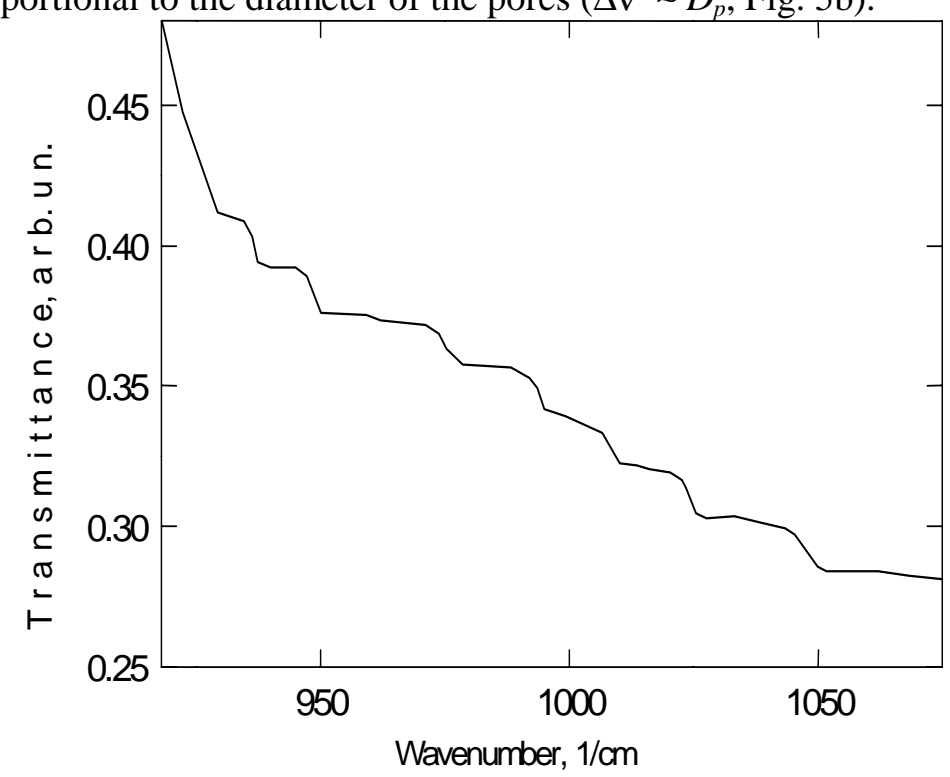

Fig. (4a): Long-wave steps in the transmittance spectrum of macroporous silicon.

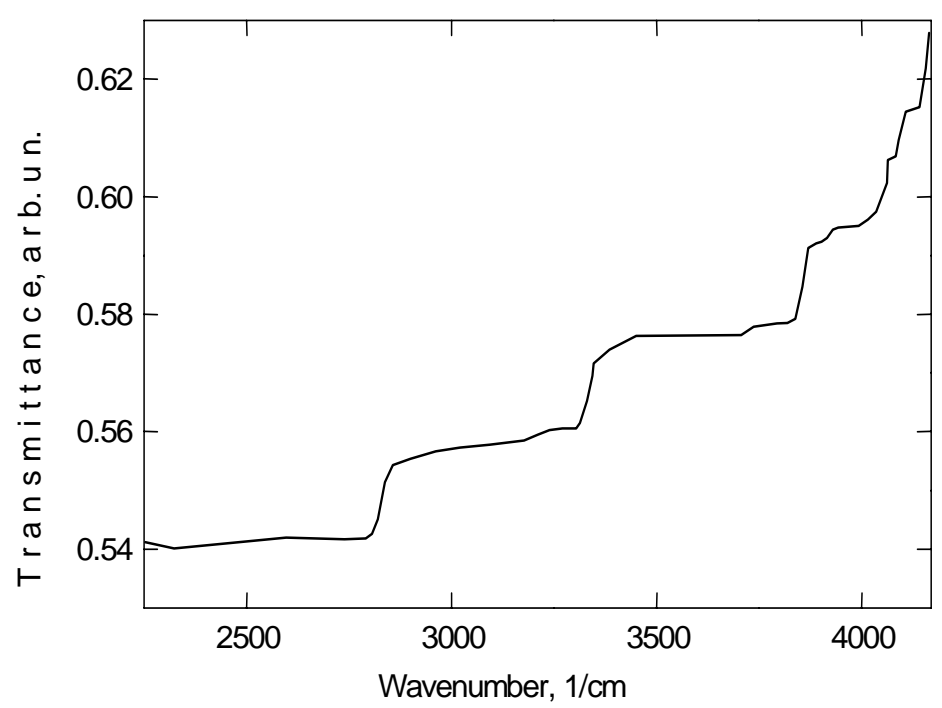

Fig. (4b): Short-wave steps in the transmittance spectrum of macroporous silicon. 

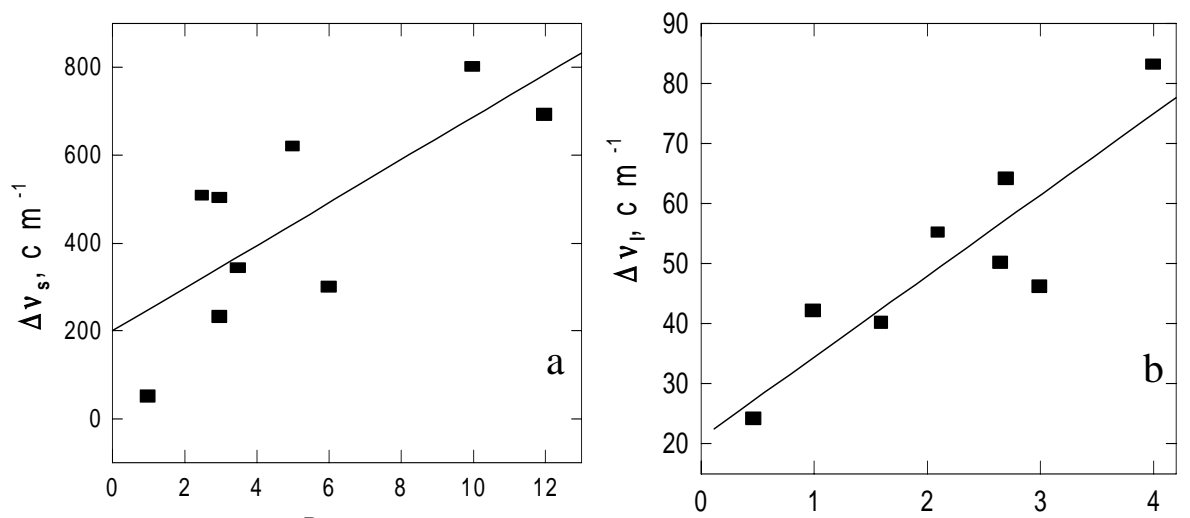

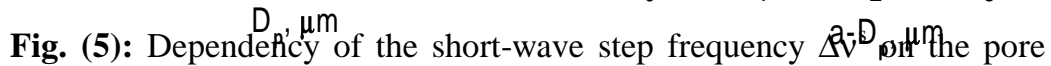
diameter $D_{p}$. (a), dependency of the long-wave step frequency $\Delta \mathrm{v}^{1}$ on the distance between pores $a-D_{p}$ (b).

Transmission spectra of macroporous silicon as well as the formation of the steps can be explained by a model of directed and radiated optical modes $[13,14]$. Macroporous silicon is a short wave-guide structure with a large contrast in dielectric constants of the pores and the silicon matrix (Fig. 6). In the short-wave spectral region the directed optical modes are formed on macropores, because the step growth of transmittance takes place for $\lambda \leq D_{p}$ (Fig. 4b). In the middle region $D_{p}<\lambda<\lambda_{a}$ the directed mode formation it is possible for the silicon wave-guides and decay mode formation for the macropores. In the longwave spectral region $\lambda>\lambda_{a}$ decay apparently $\mathrm{Si}$ matrix modes are formed [15].
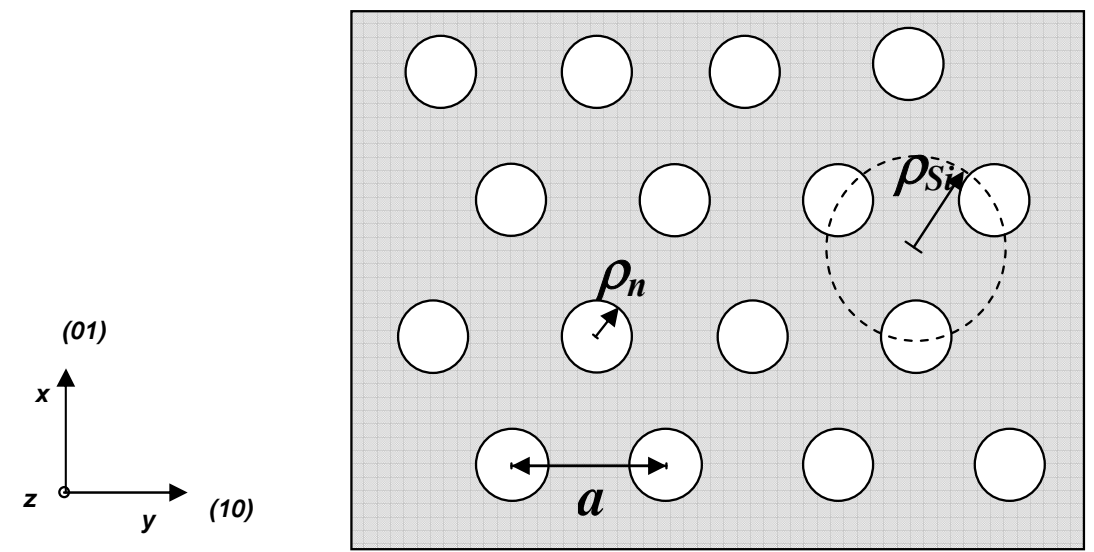

Fig. (6): Cross section of macroporous silicon structure with pore radius $\rho_{p}$ and radius of wave-guide on silicon matrix $\rho_{S i}$. 
The increase in the diameter of macropores up to the value $D_{p}>\left(a-D_{p}\right) n_{S i}$ modifies the transmission spectrum. In this case the region of the decay mode formation corresponds to $\lambda>D_{p}$, and the directed modes are formed for $\lambda<a$ $D_{p}$. Thus, in contrast to the structures with pore diameters $D_{p} \sim\left(a-D_{p}\right) n_{S i}$ for samples with large macropore diameters the decay modes are formed on pores, and the directed modes are formed on the silicon matrix. For structures with small pore diameters $\left(D_{p} \approx 1 \mu \mathrm{m}\right)$ the transmission spectrum oscillations in the region of wavelengths $\lambda<\lambda_{a}$ are measured only.

Transmission spectra of macroporous silicon structures were measured by spectrophotometer with the aperture about $10^{\circ}$, therefore at formation of optical mode the multimode regime should be realized. However, the step formation testifies to realization the one mode regime that is connected with surface oscillator fluctuations on a macropore surface and with the surface polariton formation. This is supported by the preferable absorption of the p-components of the incident electromagnetic radiation macroporous silicon structures (Fig. 7). Besides that the excitation of a surface electromagnetic wave is accompanied by the reduction in the intensity of reflected light and increase in the absorption.

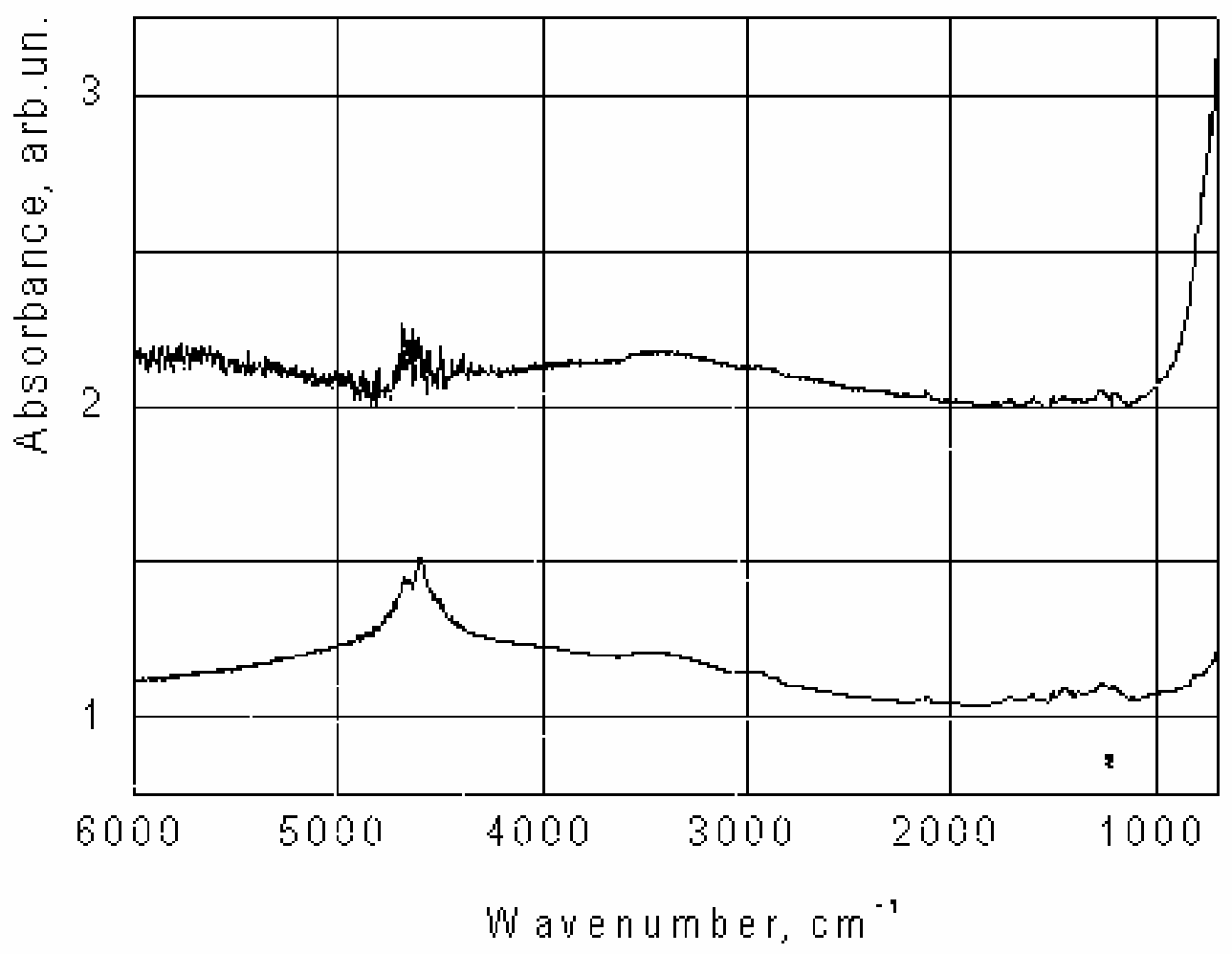

Fig. (7): Absorption spectra of the p-component (1) and s-component (2) of the incident electromaonetic radiation of macronnornus cilicon structure 


\subsection{Photoconductivity.}

Photoconductivity value depends on an angle of incidence of electromagnetic radiation (Fig. 8). It was measured in structures of macroporous silicon. For periodic structure maxima of photoconductivity are formed (1) at normal incidence of electromagnetic radiation, (2) in the region of the angle of full internal reflection respective to the macropore walls and (3) grazing incidence of light respective to the surface of the structure. At the angles of the incident light close to normal, the directed optical modes (Fig. 5) are formed on macropores. At the angle of full internal reflection respective to the macropore walls the surface TM-wave, which propagates along macropore, is formed. That is supported by the preferable absorption of the p-components of incident electromagnetic radiation by the structures of macroporous silicon (Fig. 8). At angles of incident close to grazing, the periodic relief of structure transforms incident light wave into surface one as the result of the $m$-order diffraction. It is necessary to note, that for structures with the arbitrary macropore distribution only the central maximum is formed.

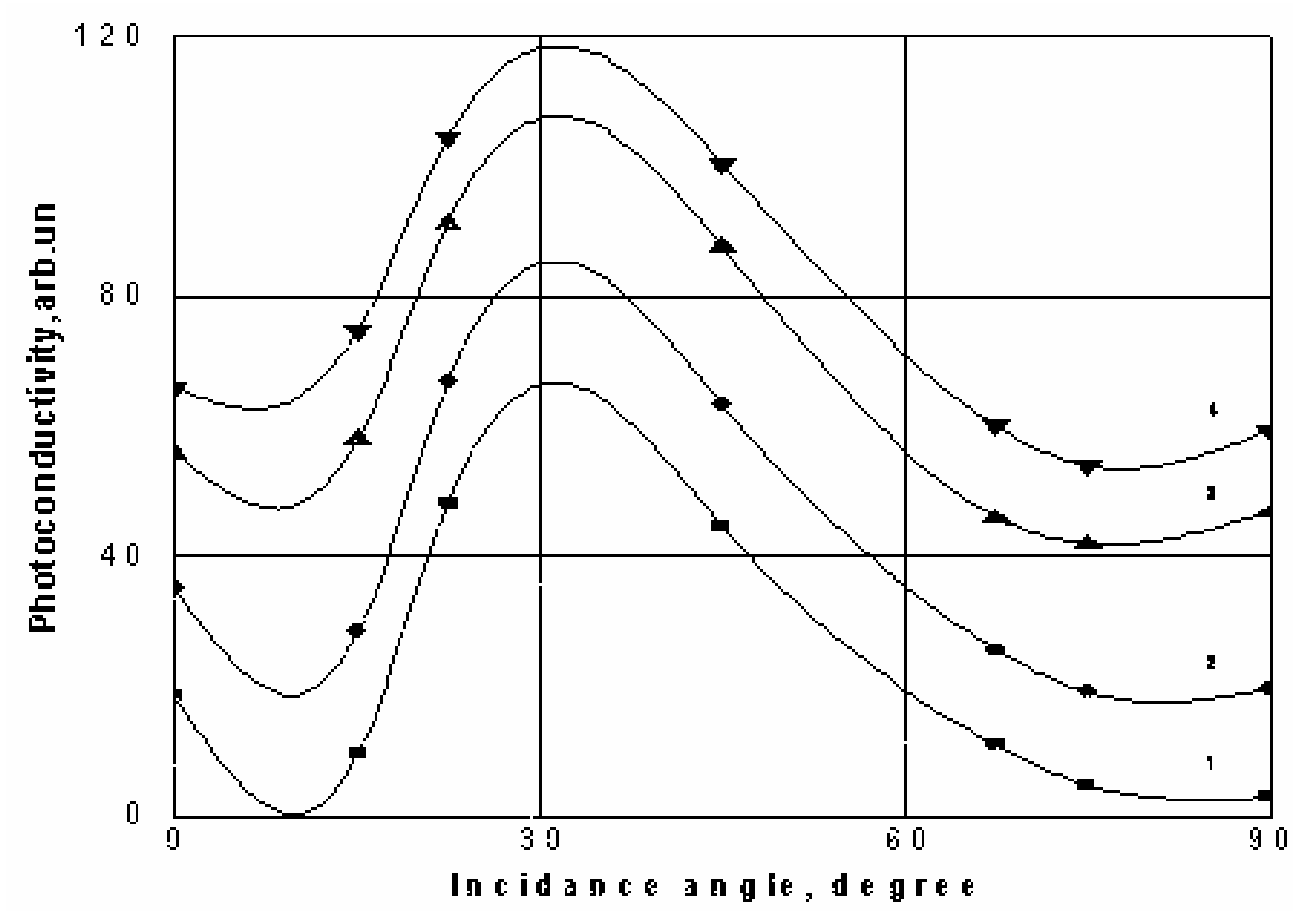

Fig. (8): Dependence of photoconductivity of the periodical macroporous silicon structure on the light incidence angle at the bias on the sample without illumination: 1 - 3 $\mathrm{mV} ; 2$ - $27 \mathrm{mV} ; 3$ - $55 \mathrm{mV} ; 4$ - 70mV ( $=77 \mathrm{~K})$ 
Surface polariton formation should result in amplification of a local electric field on a surface of structure of macroporous silicon and a macropore surface as a result of accumulation of the electromagnetic energy surpassing energy of the falling electromagnetic wave in $\left(\varepsilon^{\prime} / \varepsilon^{\prime \prime}\right)^{2}$ times, where $\varepsilon^{\prime}$ and $\varepsilon^{\prime \prime}$, accordingly, are the real and imaginary parts of dielectric permeability. Thus the dipole momentum of macropore and and its surface charge increase resonantly. For periodic macroporous structure interaction of the dipole local fluctuations is resonant too. Elecrtroreflectance spectroscopy of macroporous silicon structures showed Franz-Keldysh oscillations which indicated the development of an intrinsic electric field due to enrichment of the space-charge region with majority carriers [16]. An intrinsic electric field near $10^{6} \mathrm{~V} / \mathrm{cm}$ appears due to positive charge built in oxide layer on the walls of the macropores.

The enhancement of the photophysical phenomena is shown in (Fig. b9a) where the spectral dependence of photoconductivity is presented in the region of band-to-band light absorption of the monocrystal silicon and structures of macroporous silicon. It can be seen from (Fig.9a), that in macroporous silicon structures the signal of photoconductivity is higher then those for a monocrystal substrate in $10^{2}$ times. At the maximal enhancement of photoconductivity (Fig. 9a, curve 4) shift of a maximum in the long-wave side is measured. Correspondance of the photoconductivity spectra of macroporous silicon structures and spectra of photoconductivity of monocrystal silicon testifies on the enrichment of a macropore surface by photocarriers and formation in this spectral region of a surface electromagnetic wave of plasmon type.

Fluctuations of a surface propagate in the semiconductor to distance of length of the electron free run $L_{e}$. Hence, the amplification of the electric component of the electromagnetic field on macropore surfaces is transferred in to the volume to distance about $L_{e}$ length (about 1 micron for used in the investigations plates of silicon). In (Fig.9b) the dependence of a maximum of a photoconductivity spectra signal (Fig. 9a) on the distance between macropores $a-D_{p}$ is presented. Indeed, the greatest value of the photoconductivity signal is measured at distance between macropores $a-D_{p}=2$ microns, corresponding to $2 L_{e}$.

Surface polariton formation should result in amplification of a local electric field on a surface of structure of macroporous silicon and a macropore surface as a result of accumulation of the electromagnetic energy surpassing energy of the falling electromagnetic wave in $\left(\varepsilon^{\prime} / \varepsilon^{\prime \prime}\right)^{2}$ times, where $\varepsilon^{\prime}$ and $\varepsilon^{\prime \prime}$, accordingly, are the real and imaginary parts of dielectric permeability. Thus the dipole momentum of macropore and and its surface charge increase resonantly. For periodic macroporous structure interaction of the dipole local fluctuations is resonant too. 


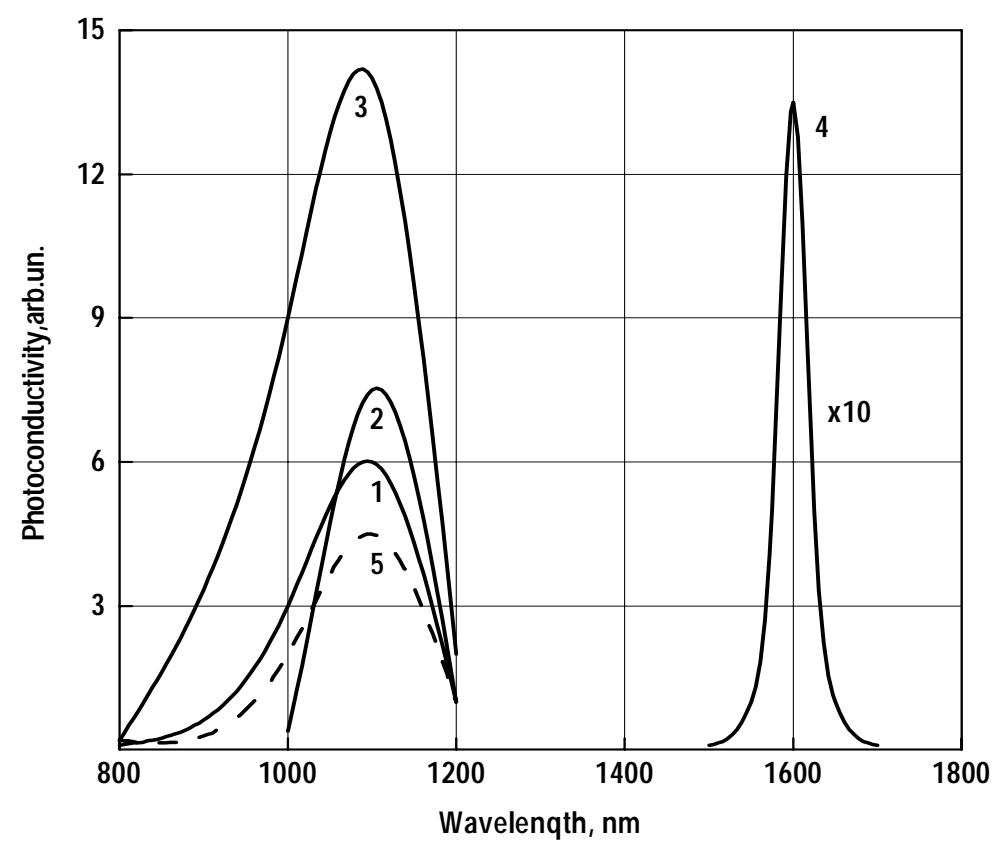

Fig. (9a): Spectral dependency of intrinsic photoconductivity of macroporous silicon

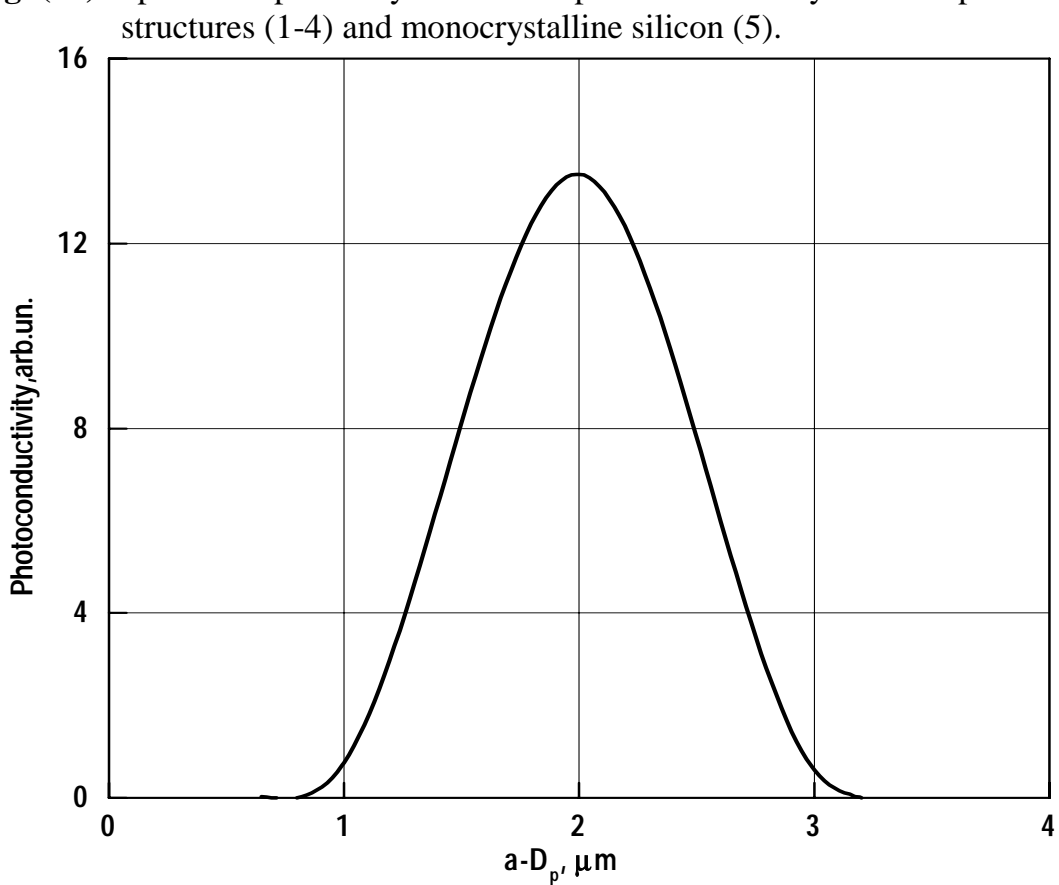

Fig. (9b): Dependency of the photoconductivity maximum on the distance between macropores $a-D_{p}$. 


\subsection{Raman spectra :}

Effects of amplification were measured also in the Raman spectra. In all Raman spectra the band was measured which corresponded to the triple degenerate vibration $\mathrm{F}_{2 \mathrm{~g}}$ of the center of Brillouin zone (Fig.10). Its position in the spectra coincided with the position of the respective band for the singlecrystalline silicon, but the intensity of the band strongly depended on the macropore sizes.

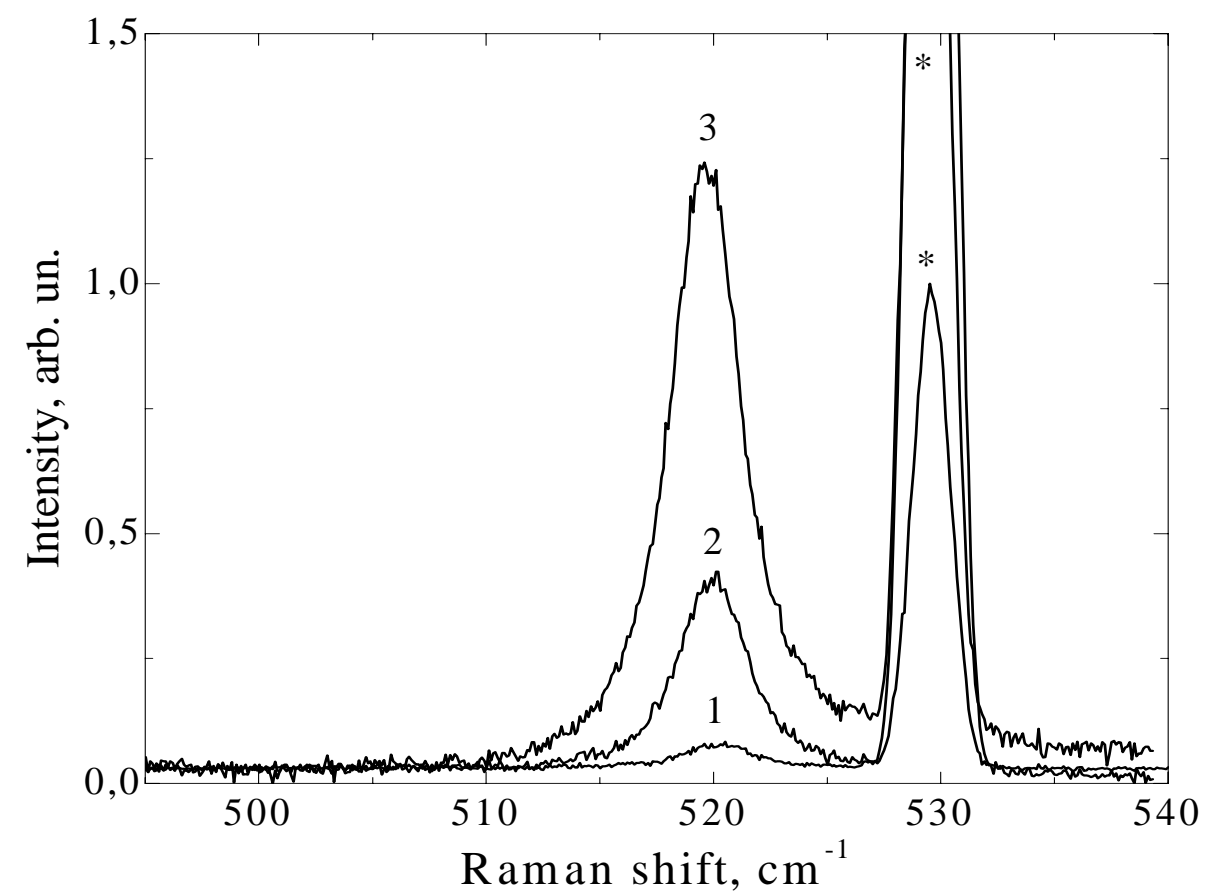

Fig. (10): Spectra of Raman scattering for macroporous silicon and silicon substrate: 1 - por-Si, with diameter of pores $10 \mu \mathrm{m}, 2$ - single-crystalline $\mathrm{Si}, 3$ por-Si, with diameter of pores $1 \mu \mathrm{m}$

Maximal scattering intensity was registered for the samples with the macropore diameter about $1 \mu \mathrm{m}$. It is necessary to note also, that for this samples scattering intensity is about 10 times bigger than for the silicon substrate. The scattering intensities ratio for the macroporous silicon and silicon substrate was maximal when the incidence angle of the laser beam was about 25-30 degrees (Fig. 11). Measurement of the maximal intensity of Raman scattering for the angle value about 25-30 degrees coincides with angle dependence of photoconductivity (Fig. 4). So the change the Raman scattering intensity with the 
change of angle may be connected with the surface polaritons formation and scattering on them. Such effect enables to investigate weak vibrations on the intensity, for example, two-phonon or $\mathrm{SiH}_{2}$ vibrations (Fig.12) and others.

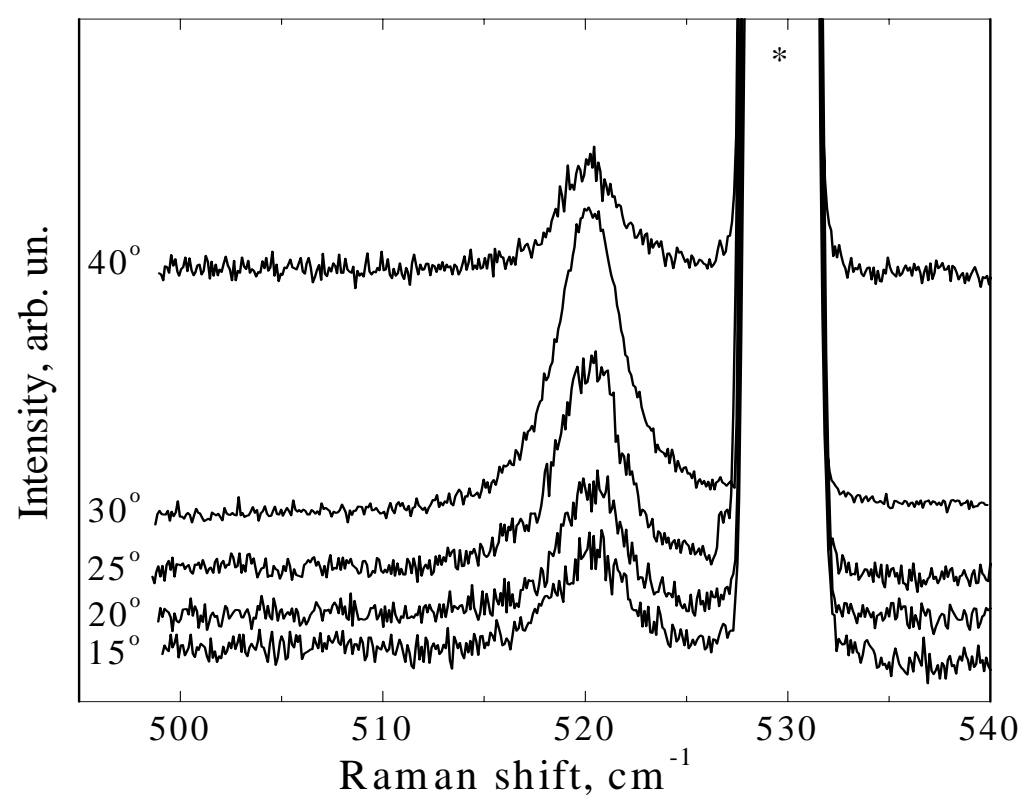

Fig. (11): Raman spectra of macroporous $\mathrm{Si}$ at different incidence angles $\theta_{\text {in }}$ of the laser beam.

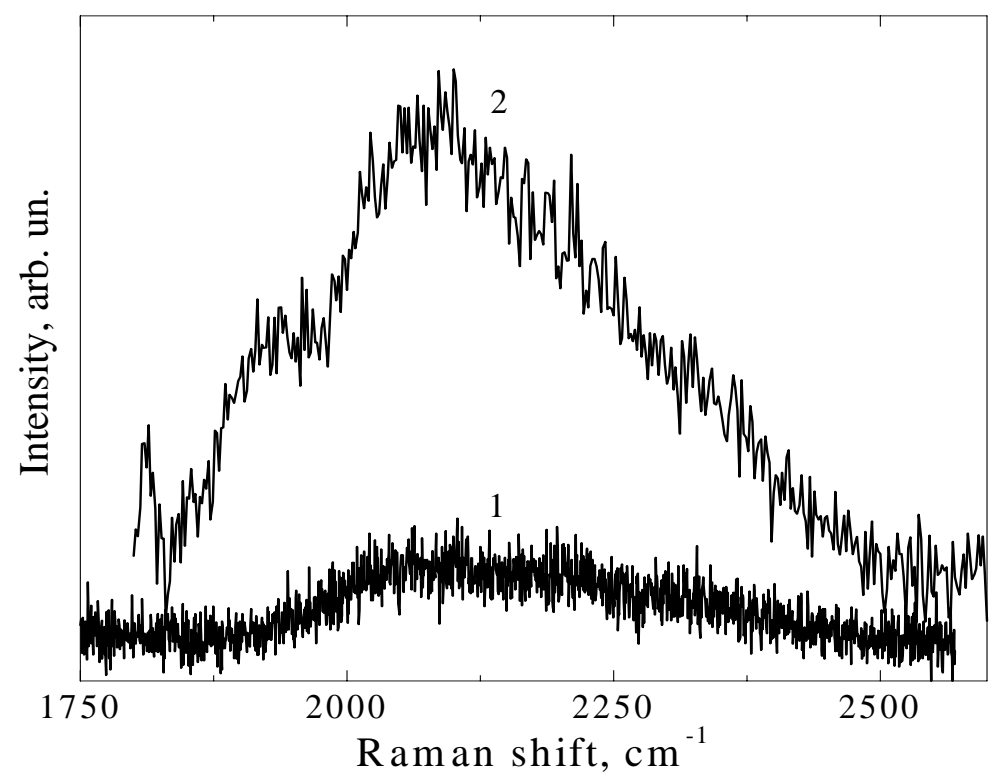

Fig. (12): Raman spectra of $\mathrm{Si}-\mathrm{H}_{2}$ vibrations in macroporous $\mathrm{Si}$ with different diameter of macropores: $-2 \mu \mathrm{m} ; 2-1 \mu \mathrm{m}$. 


\section{Conclusions:}

Effects of increase in absorption of electromagnetic radiation, amplification of photoconductivity by 2D structures of macroporous silicon were investigated. Realization of a one mode regime, preferable absorption of the pcomponents of the electromagnetic radiation are connected with interaction of optical modes with fluctuations of oscillators on a macropore surface and with surface polariton formation.

Absolute photonic band gap was measured for wavelengths between one and two optical periods of macroporous silicon structure in optical transmittance by $2 \mathrm{D}$ macroporous silicon structures. For the wavelength less than optical period of macropores there is an essential reduction in transmittance of electromagnetic radiation to (3-6) $\times 10^{-3}$ in comparison with the homogeneous material and the step formation.

Dependence of photoconductivity on the angle of incidence of the electromagnetic radiation is observed with maximum at normal incidence of electromagnetic radiation, in the region of the angle of full internal reflection respective to macropore walls and at a grazing angle of incidence respective to the surface of structure. The absolute maximum of photoconductivity is measured at distance between macropores, corresponding to two lengths of the electron free run, i.e. by the maximal transfer of the amplified electric components from a macropore surface in a silicon matrix.

Enhancement of a local electric field on macropores increases effective coefficient of absorption by macroporous silicon structure 103 times, the signal of photoconductivity amplifies $10^{2}$ times, Raman scattering - up to one order of value.

\section{Aknowledgments:}

I would like to thank Prof. M. Valakh, Dr. V. Yukhymchuk for Raman spectra investigations and my collaborators for assistance in experiments and fruitful discussion. 


\section{References:}

1. E. Yablonovitch, Phys. Rev. Lett. 58 (20), 2059 (1987).

2. S. John, Phys. Rev. Lett. 58(23), 2486(1987).

3. M. Dmitruk, V. Litovchenko, V. Strizshevsky, Surface Polaritons in Semiconductors and Dielectrics, , Kyiv, Naukova Dumka, 1989, p. 376.

4. E. Venger, A. Goncharenko M. Dmitruk, Optics of Small Particles and Disperse Media, Kyiv, Naukova Dumka, 1999, p. 348.

5. L.A. Karachevtseva, O.A. Lytvynenko, E.A. Malovichko, Theoretical and experimental chemistry (Rus.) 34 (5), 314 (1998).

6. L.A. Karachevtseva, O.A. Lytvynenko, E.A. Malovichko, E.I Stronskaya, Theoretical and experimental chemistry (Rus.) 36 (3), 193 (2000).

7. E.H. Hroderic, Metal-Semiconductor Contacts, Clarendon, Oxford, 1978.

8. S.P. Zimin, E.P. Mosquitoes, J. Technical Physics Letters, 24(6), 45 (1998).

9. A. Maradudin, A.R. McGurn, J. Mod. Opt. 41 (2), 275 (1994).

10. M.M. Sigalas, R. Bismas, K.M. Ho, and Soukoulis, Phys. Rev. B,58(11), 6791 (1998).

11. U.Gruning and V. Lehmann, article in Photonic Band Gap Materials, Eds. C. M. Soukoulis, , Kluwer Academic Publishers, 437 (1996).

12. L.A. Karachevtseva, O.A. Lytvynenko, and E.J. Stronska, Semiconductor Physics, Quantum Electronics \& Optoelectronics 3, 22 (2000).

13. A.W.Snyder, J.D.Love, Optical Waveguide Theory, London, New York, Charman and Hall, 1983, p. 656.

14. S. Solimeno, B. Crosignani, P. DiPorto, Guiding, Diffraction and Confinement of Optical Radiation. Academic Press, New York, 1986, p. 664.

15. L. Karachevtseva, O. Lytvynenko, E. Malovichko, O. Stronska., E. Buzaneva, A. Gorchinsky, Semiconductor Physics, Quantum Electronics \& Optoelectronics, 4 (4), 347 (2001).

16. R.Yu.Holiney, L.A.Matveeva, E.F.Venger, O.A.Litvinenko, and L.A.Karachevtseva, Appl. Surface Sci. 172(3), 214 (2001). 